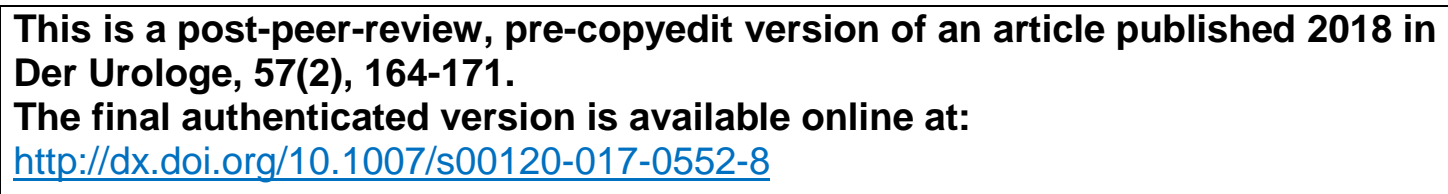

\title{
Evaluation eines Online-Tutorials zum Prostatakarzinom
}

Entwicklung und Überprüfung der Seite prostata-information.ch

\section{Autoren}

R. Schaffert ${ }^{1}$; U. Dahinden²; T. Hess²; A. Bänziger ${ }^{1}$; P. Kuntschik²; F. Odoni²; P. Spörri3;

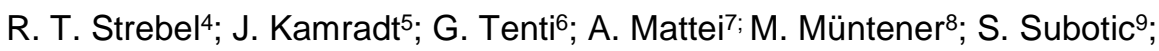

H.-P. Schmid ${ }^{10}$; P. Rüesch ${ }^{1}$

1 Institut für Gesundheitswissenschaften, Zürcher Hochschule für Angewandte Wissenschaften

2 Departement für Angewandte Zukunftstechnologien, HTW Chur

3 Urologisches Kompetenzzentrum soH, Kantonsspital Olten

4 Urologie, Kantonsspital Graubünden

5 Zentrum für Urologie und Nephrologie Bern

6 Urologische Klinik, Kantonsspital Münsterlingen

7 Klinik für Urologie, Luzerner Kantonsspital

8 Urologische Klinik, Stadtspital Triemli Zürich

9 Urologische Universitätsklinik Basel, Kantonsspital Baselland

10 Klinik für Urologie, Kantonsspital St.Gallen

\section{Korrespondenzadresse}

René Schaffert

Forschungsstelle Gesundheitswissenschaften

Departement Gesundheit

Zürcher Hochschule für Angewandte Wissenschaften

Technikumstrasse 81, CH-8401 Winterthur

rene.schaffert@zhaw.ch 


\section{Zusammenfassung}

\section{Hintergrund}

Angesichts verschiedener Behandlungsoptionen ist die Information und Therapieentscheidung beim lokalisierten Prostatakarzinom eine Herausforderung. Die digitale Informationstechnologie bietet im Vergleich zu gedruckten Informationen mehr Möglichkeiten, die Information und die Patientenkommunikation bedarfsgerecht zu gestalten.

\section{Ziele}

Zur Unterstützung der Therapieentscheidung und der Kommunikation mit Patienten ist in der deutschsprachigen Schweiz ein Online-Tutorial in einem systematischen Prozess entwickelt und in einer Pilotstudie getestet worden. In der Evaluation interessierten die Nutzerzufriedenheit, die Erfüllung der Informationsbedürfnisse, die Vorbereitung auf die Therapieentscheidung und deren subjektive Qualität.

\section{Material und Methoden}

Die Plattform wurde in einem iterativen Prozess mittels Fokusgruppen mit Ärzten und Patienten auf der Grundlage von Informationen aus bestehenden Broschüren entwickelt. Für den Test der Plattform wurden in 8 urologischen Kliniken 87 Patienten zur Teilnahme eingeladen. Die 56 Nutzer wurden 4 Wochen nach dem Login und 3 Monate nach dem Therapieentscheid online befragt, 48 Nutzer füllten beide Befragungen aus. Eingesetzte Instrumente waren die Preparation for Decision Making Scale (PDMS), die Decisional Conflict Scale (DCS) und die Decisional Regret Scale (DRS).

\section{Ergebnisse und Diskussion}

Die Nutzenden sind mit der Plattform sehr zufrieden und finden ihre Informationsbedürfnisse gut erfüllt. Sie zeigen 3 Monate nach dem Entscheid eine gute Vorbereitung auf die Entscheidung (MW PDMS 75, SD 23) und berichten über niedrigen Entscheidungskonflikt (MW DCS 9.6, SD 11) und kaum Bedauern über die Entscheidung (MW DRS 6.4, SD 9.6). Basierend auf diesen Erkenntnissen kann die Plattform zur weiteren Nutzung empfohlen werden.

\section{Schlüsselwörter}

Prostatakarzinom, Patienteninformation, Entscheidungsfindung, Internet, Versorgungsforschung 


\title{
Evaluation of a Prostate Cancer E-Health-Tutorial
}

Development and testing of the website prostata-information.ch

\begin{abstract}
Background

Due to the multitude of therapy options the treatment decision after diagnosis of a localised prostate cancer is challenging. Compared to printed booklets, web based information technology offers more possibilities to tailor information to patients' individual needs.
\end{abstract}

\section{Objectives}

To support the decision making process as well as the communication with patients we developed an online tutorial in a systematic process in the German speaking part of Switzerland and then tested it in a pilot study. The study investigated users' satisfaction, the coverage of information needs, the preparation for decision making and the subjective quality of the decision.

\section{Materials and methods}

Based on already existing information material the online tutorial was developed in an iterative process using focus groups with patients and urologists. For the following evaluation in eight clinics a total of 87 patients were invited to access the platform and participate in the study. From these patients 56 used the tutorial and 48 answered both surveys (the first one 4 weeks after the first login and the second one 3 months after treatment decision). The surveys used the Preparation for Decision Making Scale (PDMS), the Decisional Conflict Scale (DCS), and the Decisional Regret Scale (DRS).

\section{Results and Conclusion}

Satisfaction with the tutorial is very high among patients with newly diagnosed localized prostate cancer. Users find their information needs sufficiently covered. Three months after the decision they felt that they were well prepared for the decision making (Mean PDMS 75, SD 23), they had low decisional conflict (Mean DCS 9.6, SD 11) and almost no decisional regret (Mean DRS 6.4, SD 9.6). Based on these findings the further use of the tutorial can be recommended.

\section{Key words}

Prostate cancer, Decision making, Patient education, Internet, Health service research 
Die Entscheidungsfindung nach der Diagnose eines lokalisierten Prostatakarzinoms ist komplex. Verschiedene Optionen mit unterschiedlichen Vor- und Nachteilen stehen zur Auswahl und Patienten haben unterschiedliche individuelle Informationsbedürfnisse. Im Vergleich zu klassischen Printbroschüren bieten Online-Applikationen vielfältigere Kommunikationsmöglichkeiten um dieser Situation gerecht zu werden. Der vorliegende Beitrag beschreibt die Entwicklung und die Evaluationsresultate eines Online-Tutorials zur Unterstützung der Entscheidungsfindung bei lokalisiertem Prostatakarzinom.

In den meisten Europäischen Ländern steht das Prostatakarzinom an erster Stelle bei den jährlichen Karzinomneuerkrankungen beim Mann [1], so auch in der Schweiz mit insgesamt 5765 Neuerkrankungen im Jahr 2013 [2]. Angesichts von unterschiedlichen Vor- und Nachteilen verschiedener Therapieoptionen und individuellen Informationsbedürfnissen von Patienten ist die Information zur Therapieentscheidung beim lokalisierten Prostatakarzinoms komplex. Es gilt neben medizinischen Aspekten auch die Werte und Präferenzen der Patienten [3] und die verschiedenen Informationsbedürfnisse [4, 5] zu beachten. Dabei konnten Urologinnen und Urologen in der Schweiz bislang Printbroschüren [6, 7] oder nach Bedarf selbst zusammengesuchte Informationsmaterialien einsetzen. Bis vor wenigen Jahren gab es im deutschsprachigen Raum noch kaum, von unabhängigen Stellen getragene, Webseiten zu Prostatakrebs, die das Potenzial der Informationstechnologie über das Anbieten einfacher textbasierter Informationen oder einen Broschüren-Download hinaus ausnutzen. Ein Vorteil der digitalen Informationsvermittlung liegt aber gerade darin, dass über die Einbindung interaktiver Elemente die Bedürfnisse der Patienten individuell bedient werden können.

Mit dem Ziel, diese Vorteile für die Information beim lokalisierten Prostatakarzinom zu nutzen, ist in der Schweiz ein von der Krebsforschung Schweiz finanziertes Projekt zur Entwicklung eines OnlineTutorials lanciert worden. Die Website wurde gezielt so aufgebaut, dass sie zu einem späteren Zeitpunkt öffentlich zugänglich gemacht werden kann und von Patienten auch ohne bereits vorhandene detaillierte Untersuchungsergebnisse nutzbar ist. Dies ist ein wichtiger Unterschied zur in Deutschland unter dem Dach der „Patienten-Akademie der Deutschen Urologen“ entwickelten Entscheidungshilfe, die durch Angaben des Patienten zu verschiedenen Parametern auf eine spezifische Situation zugeschnitten wird [8].

\section{Fragestellung}

In diesem Beitrag wird die Entwicklung des Schweizer Online-Tutorials „www.prostata-information.ch“ (Benutzername: visitor; Passwort: guest2017) vorgestellt und auf die Ergebnisse einer Pilotstudie zur Evaluation eingegangen. In der Pilotstudie interessierte, wie die Patienten das Online-Tutorials nutzen und beurteilen, inwieweit subjektive Informationsbedürfnisse abgedeckt sind, wie gut die Website auf die Entscheidung vorbereitet und wie gut die subjektive Entscheidungsqualität ist. 


\section{Studiendesign und Untersuchungsmethoden}

\section{Entwicklung des Tutorials}

Die Website wurde in einem nutzerzentrierten Ansatz mittels eines iterativen Software-

Entwicklungsprozesses in verschiedenen Schritten nach Vorgaben der International Organisation for Standardisation entwickelt [9]. Aufbauend auf eine Literatur- und Online-Recherche und explorative Interviews mit Patienten und Urologen $(\mathrm{N}=4)$ wurde die Informationsarchitektur des Tutorials festgelegt. Die Inhalte wurden auf der Grundlage von bereits bestehenden, von Fachleuten erarbeiteten, Informationsbroschüren aus der Schweiz und Deutschland erarbeitet $[6,7,10]$ und in einem schriftlichen Feedbackprozess von in die Studie involvierten Urologen geprüft. Damit konnte die Forderung des HON Code of Conduct for medical and Health Websites [11] erfüllt werden, dass abgegebene Informationen von medizinisch ausgebildeten und qualifizierten Personen stammen müssen. Die Erarbeitung erfolgte in zwei Durchläufen über Entwicklung, Evaluation und Anpassung wobei je zwei Fokusgruppen mit den gleichen Patienten $(\mathrm{N}=5)$ und Urologen $(\mathrm{N}=6)$ durchgeführt wurden. Abschliessend erfolgte im Rahmen eines Pilottests eine quantitative Überprüfung bei Patienten.

\section{Rekrutierung und Design des Pilottests}

Für den Test des Tutorials wurden in acht urologische Kliniken in der Deutschschweiz Patienten mit diagnostiziertem lokalisiertem Prostatakarzinom rekrutiert. Die Information zur Studie erfolgte mündlich und mittels schriftlichen Studieninformationen im Rahmen einer Konsultation, in der Therapieoptionen nach erfolgter Diagnose diskutiert wurden. Patienten erhielten eine Einverständniserklärung und einen Zugangscode zur Website und konnten diese anschliessend zur Vertiefung und Ausweitung der Information und zur Vorbereitung auf eine nächste Konsultation nutzen. Folgende Einschlusskriterien wurden angewandt: Tumorstadium T1 oder T2, PSA $<20 \mathrm{ng} / \mathrm{ml}$, Gleason-Score $<8$, Alter $\leq 75$, vermutete Lebenserwartung $>10$ Jahre. Ausschlusskriterien waren: mangelnde Sprachkenntnisse, mangelnde Urteilsfähigkeit und besondere emotionalen Problemlagen. Weiter mussten die Teilnehmenden über ein internetfähiges Gerät verfügen und basale Bedienungskenntnisse haben. Die Studie wurde von den zuständigen Ethikkommissionen geprüft und bewilligt (ID: KEK-ZH Nr. 2015-0132).

Patienten, die sich in die Webseite eingeloggt und die Einverständniserklärung unterzeichnet hatten, wurden zu zwei Zeitpunkten zu einer Online-Befragung eingeladen. Die Erstbefragung erfolgte vier Wochen nach dem ersten Einloggen und enthielt Fragen zur Benutzung und Beurteilung der Webseite zur bevorzugten Therapie, zur Vorbereitung auf die Entscheidung mittels der Preparation for Decision Making Scale [12], zur Erhebung des Entscheidungskonfliktes mittels der Decisional Conflict Scale [13] sowie zur Soziodemografie.

Die zweite Befragung erfolgte drei Monate nach dem Datum des Therapieentscheides und enthielt Fragen zur Befriedigung der subjektiven Informationsbedürfnisse, die mittels eines angepassten Instruments von Boberg et al. [14] erfasst wurde. Ebenfalls erfasst wurden die subjektive Entscheidungsqualität mit der Decisional Regret Scale [15]. 


\section{Datengrundlage und Analysen}

Während 15 Monaten sind in den beteiligten Kliniken Patienten zur Nutzung des Tutorials und zur Teilnahme an der Studie eingeladen worden. Von 87 mit Studieninformationen versorgten Patienten haben sich 56 (64\%) eingeloggt und $53(61 \%)$ haben an der ersten Befragung teilgenommen. Insgesamt 48 Patienten beantworteten auch die zweite Befragung vollständig, was zu einer gesamthaften Teilnahmequote von $56 \%$ führt.

Die Auswertungen erfolgten mit der Statistiksoftware IBM SPSS 24 und sind primär deskriptiver Natur.

\section{Ergebnisse}

\section{Gestaltungselemente des Tutorials}

Die Webseite „prostata-information.ch“ ist so gestaltet, dass Nutzer abhängig von ihren individuellen Informationsbedürfnissen direkt mittels Anklicken eines Themenbereichs zu den gewünschten Informationen gelangen (s. Abb. 1). Durch das mögliche Aufklappen von zusätzlichen, initial verdeckten, Informationen und das Bereitstellen eines Glossars wird der unterschiedlichen gewünschten Informationstiefe begegnet (s. Abb. 2). Für die visuelle Informationsvermittlung sind rund 50 Bilder und Informationsgrafiken erstellt worden. Ein mit Fragen zu zentralen Informationsbereichen strukturierter digitaler Notizblock kann ausgedruckt werden und zu einer nächsten Konsultation mit dem behandelnden Arzt oder der behandelnden Ärztin mitgebracht werden (s. Abb. 3).

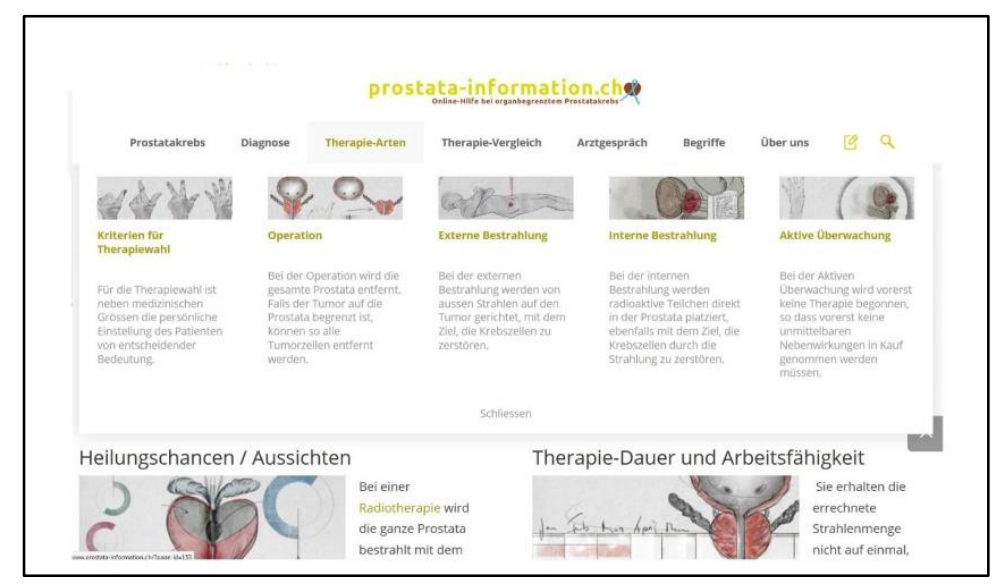

Abb. 1: Auszug aus dem Online-Tutorial: Übersicht Gastzugang für Leser: www.prostata-information.ch Benutzername: visitor Passwort: guest2017

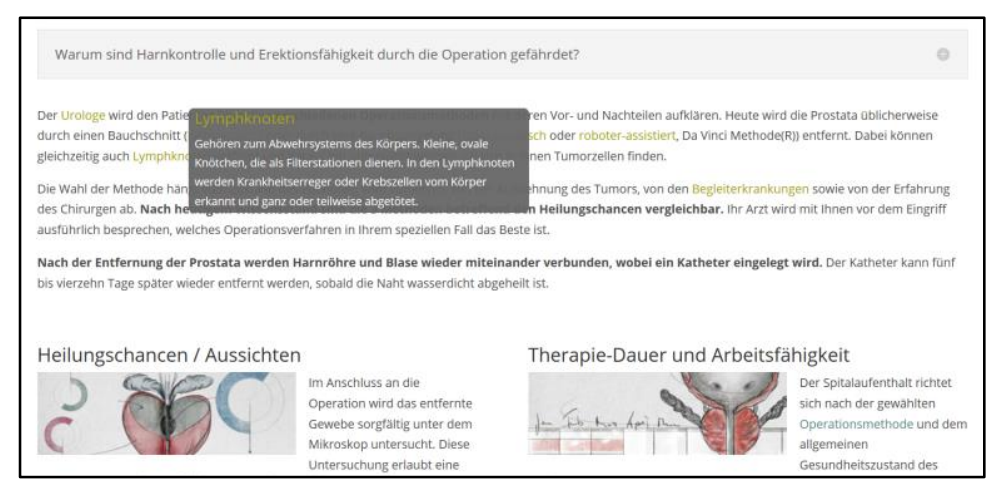

Abb. 2: Auszug aus dem Online-Tutorial: Unterschiedliche Informationstiefe 


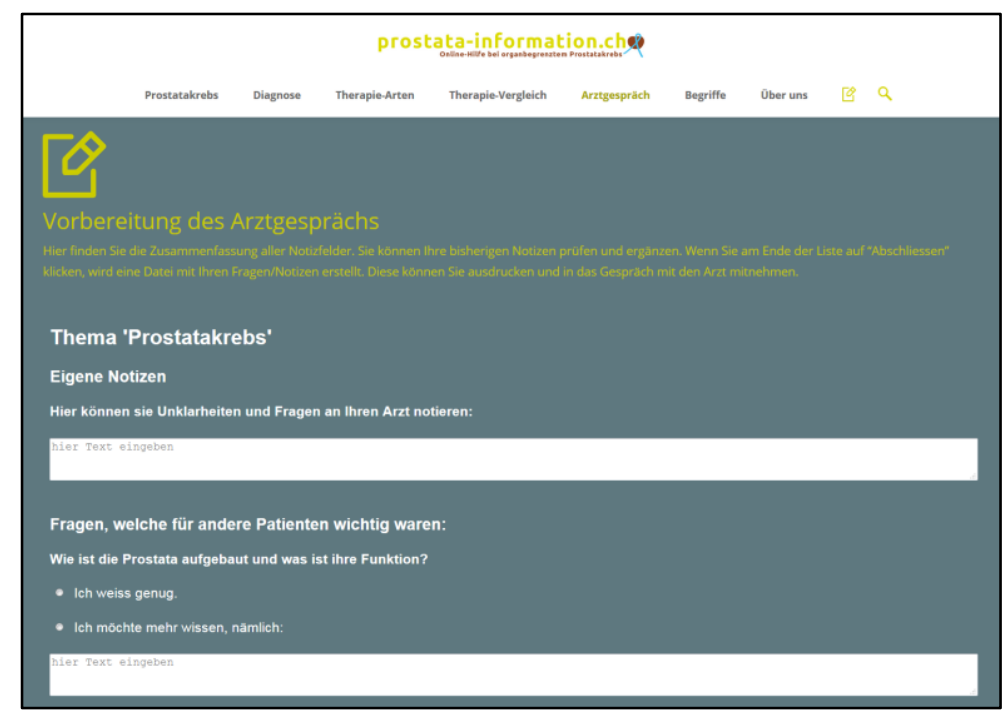

Abb. 3: Auszug aus dem Online-Tutorial: Digitaler Notizblock

\section{Resultate der Befragungen}

Die Studienteilnehmer sind zwischen 50 und 75 Jahren alt (Mittelwert 64). Die Hälfte ist noch berufstätig und alle haben mindestens einen Abschluss auf Sekundarstufe (Berufslehre oder Mittelschule), eine knappe Mehrheit von 55\% hat einen Tertiärabschluss (Höhere Fachschulen oder Universität).

Zum Zeitpunkt der Erstbefragung präferiert eine Mehrheit von 58\% der Patienten eine radikale Prostataektomie, 15\% eine Brachytherapie, 10\% eine perkutane Radiotherapie und ebenfalls $10 \%$ eine aktive Überwachung. Rund 7\% der Patienten sind zu diesem Zeitpunkt noch unsicher. Der Vergleich mit den anschliessend umgesetzten Therapien zeigt, dass diese Präferenzen von keinem Patienten umgestossen wird und die noch unsicheren Patienten sich entscheiden können.

Die Teilnehmenden nutzen das Tutorial gemäss der Auswertung von Fragen zur spezifischen Nutzung vor der Erstbefragung unterschiedlich intensiv: Ein Anteil von 28\% besucht die Seite nur einmal, eine Mehrheit von 55\% zwischen zwei und fünf Mal und 13\% tun dies mehr als fünf Mal. Die durchschnittliche Nutzungszeit über aller Seitenbesuche beträgt 2.4 Stunden (Median 2; SD 2.46) wobei sich grosse Unterschiede von zwischen 15 Minuten und 15 Stunden zeigen. Etwas mehr als drei Viertel besucht die Webseite zwischen der Erstbefragung und der drei Monate nach Therapieentscheid angesetzten Zweitbefragung erneut.

Die Teilnehmenden bewerten das Tutorial anhand von 7 Beurteilungskriterien mit einer Zustimmungsquote von $77 \%$ bis $98 \%$ sehr positiv (vgl. Tab. 1) und sehen sich durch das Tutorial auch gut auf die Entscheidung vorbereitet. In der diesen Aspekt messenden Preparation for Decision Making Scale zeigt sich ein Mittelwert von 75 (Median 81, Skala 0-100). In den drei gezielt auf die Kommunikation mit dem Arzt oder der Ärztin fokussierten Fragen antworten zwischen 63\% und 75\% zustimmend (s. Tab. 2 und 3). 


\begin{tabular}{|c|c|c|c|c|}
\hline $\begin{array}{l}\text { Bitte geben Sie an, wie sehr die folgenden Aussagen in Bezug auf die } \\
\text { Online-Plattform zutreffend sind. Die Online-Plattform ... } \\
\text { Trifft vollständig zu (1); Trifft eher zu (2); Teils-teils (3); Trifft eher nicht zu (4); } \\
\text { Trifft überhaupt nicht zu (5); Kann ich nicht beantworten (missing) }\end{array}$ & $\begin{array}{c}\text { Anteil } \\
\text { Zustimmung }\end{array}$ & MW & SD & $\mathrm{N}$ \\
\hline ... enthält verständliche Texte. & $96 \%$ & 1.3 & 0.54 & 51 \\
\hline ... fasst die wichtigsten Informationen kurz zusammen. & $98 \%$ & 1.3 & 0.51 & 51 \\
\hline ... bietet genügend ausführliche Informationen. & $88 \%$ & 1.5 & 0.70 & 51 \\
\hline ... enthält hilfreiche Abbildungen. & $94 \%$ & 1.4 & 0.66 & 51 \\
\hline ... enthält neutrale Informationen, die keine Therapieform bevorzugen. & $92 \%$ & 1.4 & 0.70 & 50 \\
\hline ... wurde von Organisationen aufgebaut, denen ich vertraue. & $96 \%$ & 1.4 & 0.58 & 45 \\
\hline ... enthält vertrauenswürdige Informationen. & $98 \%$ & 1.3 & 0.51 & 50 \\
\hline ... hat mir neues Wissen vermittelt. & $86 \%$ & 1.6 & 0.90 & 51 \\
\hline ... ist einfach zu benutzen. & $96 \%$ & 1.3 & 0.54 & 51 \\
\hline ... macht Freude bei der Benutzung. & $77 \%$ & 1.9 & 0.95 & 44 \\
\hline ... würde ich anderen empfehlen. & $96 \%$ & 1.2 & 0.50 & 51 \\
\hline
\end{tabular}

\begin{tabular}{|c|c|c|c|c|c|}
\hline $\begin{array}{l}\text { Bitte geben Sie an, wie sehr die folgenden Aussagen in Bezug auf } \\
\text { die Online-Plattform zutreffend sind. } \\
\text { Die Online-Plattform hat ... } \\
\text { Trifft vollständig zu (5); Trift eher zu (4); Teils-teils (3); Trifft eher nicht zu (2); } \\
\text { Trifft überhaupt nicht zu (1); }\end{array}$ & MED & MW & $\begin{array}{l}\text { Anteil eher / } \\
\text { vollständig } \\
\text { zutreffend }\end{array}$ & SD & $\mathrm{N}$ \\
\hline ... mir geholfen, auf Fragen zu kommen, die ich dem Arzt stellen will. & 4 & 3.85 & $63 \%$ & 1.10 & 46 \\
\hline $\begin{array}{l}\text {... mich darauf vorbereitet, mit meinem Arzt darüber zu sprechen, } \\
\text { was mir am wichtigsten ist. }\end{array}$ & 4 & 3.89 & $75 \%$ & 1.22 & 47 \\
\hline $\begin{array}{l}\text {... mir geholfen, mich auf den nächsten Termin mit meinem Arzt } \\
\text { vorzubereiten. }\end{array}$ & 4 & 4.00 & $75 \%$ & 1.19 & 48 \\
\hline
\end{tabular}

\begin{tabular}{|c|c|c|c|c|c|c|c|}
\hline Skala oder Subskala & $\begin{array}{l}\text { Anteil mind. } \\
25 \text { Punkte }\end{array}$ & $\begin{array}{l}\text { Anteil min. } \\
\text { Ausprägung }\end{array}$ & MED & MW & Max. & SD & $\mathrm{N}$ \\
\hline Vorbereitung: Preparation for Decision Making & * & * & 81 & 75 & 100 & 23.13 & 44 \\
\hline Entscheidungskonflikt: DCS Gesamt-Score & $11 \%$ & $15 \%$ & 6.25 & 9.6 & 41 & 10.96 & 47 \\
\hline - DCS Unterskala: Unsicherheit & $29 \%$ & $44 \%$ & 8.3 & 13.6 & 67 & 16.99 & 52 \\
\hline - DCS Unterskala: Information & $14 \%$ & $58 \%$ & 0 & 8.2 & 42 & 11.72 & 52 \\
\hline - DCS Unterskala: Werteklarheit & $23 \%$ & $60 \%$ & 0 & 10.1 & 50 & 14.97 & 52 \\
\hline - DCS Unterskala: Unterstützung & $20 \%$ & $50 \%$ & 4.2 & 10.2 & 50 & 13.18 & 52 \\
\hline - DCS Unterskala: Effektive Entscheidung & $10 \%$ & $56 \%$ & 0 & 6.4 & 37.5 & 9.63 & 52 \\
\hline Beurteilung Entscheid: Decisional Regret Scale & $9 \%$ & $59 \%$ & 0 & 6.4 & 42 & 11.01 & 46 \\
\hline \multicolumn{8}{|c|}{$\begin{array}{l}\text { MED=Median; MW=arithmetisches Mittel; SD=Standardabweichung } \\
\text { "keine sinnvolle Angabe, da hohe Ausprägungen positiv sind im Gegensatz zu anderen Skalen. } \\
\text { Entscheidungskonflikt: Decisional Conflict Scale, Skala von } 0 \text { bis } 100 \text { von keinem bis ausgeprägtem Konflikt (Werte über } 25 \\
\text { entsprechen einem klinisch relevanten Konflikt; Referenzen s. Text) } \\
\text { Beurteilung des Entscheids: Decisional Regret Scale, Skala von } 0=\text { kein Bedauern bis } 100=\text { grosses Bedauern } \\
\text { Vorbereitung zur Entscheidung mittels Tutorial: Preparation for Decision Making: Skala von } 0 \text { bis } 100\end{array}$} \\
\hline
\end{tabular}

Vier Wochen nach der erstmaligen Nutzung des Tutorials haben die Teilnehmenden nach erfolgter Information in der Konsultation und nach der zeitweiligen Nutzung des Tutorials kaum Entscheidungskonflikte. Sie zeigen einen Mittelwert von 9.6 (Median 6) auf der entsprechenden bis 100 reichenden Skala und rund $11 \%$ erreichen oder überschreiten den als klinisch relevant eingestuften Wert von 25 [16] (s. Tab. 3).

Drei Monate nach dem Therapieentscheid fühlen sich die Teilnehmenden anhand des ganzen Informationsprozesses, in dem neben dem Tutorial und den Gesprächen in Konsultationen auch andere Quellen genutzt werden können, gut informiert: In 11 von 16 Themenbereichen schätzt über die Hälfte der Teilnehmenden ihren Informationsstand als sehr gut ein. Auch die individuellen 
Informationsbedürfnisse werden mehrheitlich gut abgedeckt. Diese werden in dieser Studie als erfüllt angesehen, wenn ein Patient den Informationsstand zu einem Thema auf einer fünf stufigen Skala gleich oder höher einstuft, als auf einer vergleichbaren Skala zur Wichtigkeit. Dies ist bei allen der 16 Themen für mindestens zwei Drittel der Teilnehmenden der Fall (s. Tab. 4).

Einen weiteren Eindruck zur subjektiven Entscheidungsqualität gibt die Decision Regret Scale. Hier zeigen $60 \%$ einen Wert von 0 auf der bis 100 reichenden Skala (Median=0), dies als Ausdruck eines minimalen nachträglichen Bedauerns des Konfliktes. Der Mittelwert liegt bei 6.4 und 10\% der Nutzer erreichen oder überschreiten den Wert von 25 und zeigen somit ein gewisses Bedauern über den Entscheid (s. Tab. 4).

\begin{tabular}{|c|c|c|c|c|c|c|c|}
\hline $\begin{array}{l}\text { Wie gut informiert fühlen Sie sich bei } \\
\text { diesem Thema? } \\
\text { sehr gut (1); eher gut (2); mittelmässig (3); } \\
\text { eher nicht (4); überhaupt nicht (5) }\end{array}$ & MED & MW & $\begin{array}{l}\text { Anteil } \\
\text { sehr } \\
\text { gut }\end{array}$ & $\begin{array}{c}\text { Anteil } \\
\text { eher gut bis } \\
\text { sehr gut }\end{array}$ & $\begin{array}{l}\text { Erfüllung: Anteil } \\
\text { Informationsstand } \\
\geq \text { Wichtigkeit }\end{array}$ & SD & $\mathrm{N}$ \\
\hline Durchführung der Therapien & 1 & 1.27 & $75 \%$ & $98 \%$ & $90 \%$ & 0.49 & 48 \\
\hline Wirkungsweise der Therapien & 1 & 1.31 & $71 \%$ & $98 \%$ & $83 \%$ & 0.51 & 48 \\
\hline Tumorstadium & 1 & 1.38 & $71 \%$ & $92 \%$ & $79 \%$ & 0.64 & 48 \\
\hline $\begin{array}{l}\text { Wann die einzelnen Therapien in Frage } \\
\text { kommen }\end{array}$ & 1 & 1.38 & $67 \%$ & $96 \%$ & $79 \%$ & 0.57 & 48 \\
\hline $\begin{array}{l}\text { Auswirkungen auf die Blasenfunktion / } \\
\text { das Wasserlösen }\end{array}$ & 1 & 1.44 & $67 \%$ & $90 \%$ & $81 \%$ & 0.68 & 48 \\
\hline PSA-Wert & 1 & 1.50 & $67 \%$ & $88 \%$ & $90 \%$ & 0.83 & 48 \\
\hline Aufbau und Funktion der Prostata & 1 & 1.42 & $63 \%$ & $96 \%$ & $88 \%$ & 0.58 & 48 \\
\hline Auswirkungen auf die Zeugungsfähigkeit & 1 & 1.77 & $63 \%$ & $81 \%$ & $94 \%$ & 1.26 & 48 \\
\hline Verhalten während der Behandlung & 1 & 1.53 & $60 \%$ & $89 \%$ & $74 \%$ & 0.75 & 47 \\
\hline Auswirkungen auf die Sexualität & 1 & 1.50 & $58 \%$ & $92 \%$ & $83 \%$ & 0.65 & 48 \\
\hline Dauer der Therapien & 1 & 1.54 & $54 \%$ & $92 \%$ & $81 \%$ & 0.65 & 48 \\
\hline $\begin{array}{l}\text { Behandlung / Linderung von } \\
\text { Nebenwirkungen der Behandlung }\end{array}$ & 2 & 1.74 & $49 \%$ & $81 \%$ & $72 \%$ & 0.87 & 47 \\
\hline $\begin{array}{l}\text { weiteren Nebenwirkungen der gewählten } \\
\text { Therapie }\end{array}$ & 2 & 1.77 & $46 \%$ & $83 \%$ & $71 \%$ & 0.88 & 48 \\
\hline weiteren Unterstützungsangeboten & 2 & 1.96 & $40 \%$ & $72 \%$ & $74 \%$ & 0.98 & 47 \\
\hline Gleason-Score & 2 & 2.00 & $36 \%$ & $74 \%$ & $70 \%$ & 0.98 & 47 \\
\hline $\begin{array}{l}\text { Entstehung und Ursachen von } \\
\text { Prostatakrebs }\end{array}$ & 2 & 2.50 & $17 \%$ & $56 \%$ & $67 \%$ & 1.07 & 48 \\
\hline
\end{tabular}

\section{Diskussion}

\section{Vergleiche mit anderen Studien}

Die genutzten etablierten Messinstrumente erlauben einen Vergleich mit anderen Studien, in denen die Nutzung von Tutorials durch Patienten mit lokalisiertem Prostatakarzinom untersucht wurde. Dabei zeigt sich, dass sich die Nutzer des Online-Tutorials durch die Webseite besser auf die Entscheidung vorbereitet fühlen (MW 75) als dies für Teilnehmer der Evaluation einer Entscheidungshilfe in Kanada der Fall ist (MW 29) [17]. Minimal höher aber auf vergleichbaren Niveau liegt in Kanada auch die Beurteilung der Entscheidung mittels der Decisional Regret Scale (MW 7.4 vs. MW 6.4). Weiter finden sich bei den Studienteilnehmern aus der Schweiz auch niedrigere Entscheidungskonflikte als bei Patienten in Studien aus Spanien und den USA (MW 9.6 vs. 25 bis 31) [18-20]. Diese Vergleiche gilt es vorsichtig zu interpretiert, da nicht überprüft werden kann, inwieweit die gefundenen Werte auch aufgrund von unterschiedlichen lokalen Informationsstrukturen, Unterschieden im Sample oder kulturellen Differenzen entstehen. Die vergleichsweise guten Werte in 
den etablierten Skalen geben aber zumindest keine Hinweise darauf, dass die positive Beurteilung des Tutorials relativiert werden müsste.

\section{Limitationen}

Im Rahmen dieser Pilotstudie ohne Kontrollgruppe kann die Wirksamkeit des Online-Tutorials nicht befriedigend überprüft werden, es ist kein Vergleich zwischen Intervention und dem Standardverfahren möglich. Weiter können Ergebnisse nicht auf alle Patienten mit lokalisiertem Prostatakarzinom übertragen werden, da aufgrund der freiwilligen Teilnahme und dem Ausschlusskriterium der ungenügenden Computerkenntnisse ein selektives Sample vorliegt. Die Erkenntnisse beziehen sich auf eine Patientengruppe mit einer gewissen Affinität für Informationstechnologien. Dies wiederspiegelt sich auch im überdurchschnittlich hohen Anteil von gut gebildeten Teilnehmenden in dieser Studie. Aufgrund des kleinen Samples konnte auch nur mit Einschränkungen überprüft werden, inwieweit es Unterschiede zwischen verschiedenen Gruppen von Patienten geben könnte. Bei der Interpretation des Bedauerns des Entscheides (Decisional Regret) muss auch beachtet werden, dass 3 Monate nach der Entscheidung allenfalls noch nicht alle positiven oder negativen Konsequenzen der Therapie eingeschätzt werden können: Die gefundenen Werte könnten sich bei einer späteren Erhebung erhöhen.

\section{Fazit zum Online-Tutorial}

Auch unter Berücksichtigung der erwähnten Einschränkungen zeichnet die Pilotstudie ein ermutigendes Bild: Die Webseite wird anhand von verschiedenen Nutzungsaspekten sehr positiv bewertet und sie vermag die individuellen Informationsbedürfnisse für die überwiegende Mehrheit der Patienten zu erfüllen. Auch die subjektive Entscheidungsqualität fällt mehrheitlich sehr gut aus: Die Nutzer zeigen meist einen niedrigen Entscheidungskonflikt, fühlen sich durch das Online-Tutorial gut auf die Entscheidungsfindung vorbereitet und sind mit wenigen Ausnahmen mit der getroffenen Entscheidung zufrieden.

Auch wenn mit der vorliegenden Studie die Wirksamkeit der entwickelten Online-Plattform im Vergleich zur Aufklärung ohne Nutzung der Website nicht nachgewiesen werden kann, lässt sich aufgrund von internationalen Studienerkenntnissen eine positive Wirkung erwarten: Das Tutorial enthält verschiedene Elemente, die für Entscheidungshilfen typisch sind, so zum Beispiel eine explizite Gegenüberstellung der Therapieoptionen nach relevanten Themen oder den Fragebogen zur Vorbereitung der Konsultation. Gemäss einer Cochrane-Review bewirken Entscheidungshilfen eine Verbesserung des Wissens über die Behandlungsoptionen, eine Reduktion des Entscheidungskonfliktes und eine höhere Klarheit von Werten und Präferenzen [21]. Basierend auf den Erkenntnissen unserer Pilotstudie und der internationalen wissenschaftlichen Evidenz kann deshalb die entwickelte Website „www.prostata-information.ch“ für eine zukünftige weitere Verbreitung empfohlen werden. 


Fazit für die Praxis
Von einer Prostatakarzinomdiagnose betroffene Patienten mit Computergrundkenntnissen beurteilten
den Einsatz einer Onlineplattform mit interaktiven Elementen zur Darreichung von Informationen und
Vorbereitung von Konsultationen positiv. Die Entwicklung, Weiterentwicklung und Nutzung von
entsprechenden Plattformen erscheint deshalb sinnvoll.

\section{Literatur}

1. Ferlay, J., et al., Cancer incidence and mortality patterns in Europe: Estimates for 40 countries in 2012. Eur J Cancer, 2013. 49(6): p. 1374-1403.

2. NICER - National Institute for Cancer Epidemiology and Registration, National statistics on cancer incidence. 2016: http://www.nicer.org/en/statistics-atlas/cancer-incidence/ [zugegriffen: 11.04.2017].

3. Kollmeier, M.A. and M.J. Zelefsky, How to select the optimal therapy for early-stage prostate cancer. Crit Rev Oncol Hematol, 2012. 84 Suppl 1: p. e6-e15.

4. Feldman-Stewart, D., et al., Information needs of early-stage prostate cancer patients: a comparison of nine countries. Radiother Oncol, 2010. 94(3): p. 328-33.

5. Schaffert, R., et al., Informationsbedürfnisse von Prostatakrebspatienten - Grosse individuelle Unterschiede nach Diagnose eines lokalisierten Prostatakarzinoms. Urologe, 2011. 50(9): p. 1089-94.

6. Broccard, N., Prostatakrebs: Prostatakarzinom; eine Information der Krebsliga für Betroffene und Angehörige. 2., überar ed. 2009, Bern: Krebsliga Schweiz.

7. Schweizerische Gesellschaft für Urologie, Prostata: Kleine Drüse - grosse Bedeutung: Informationen über die häufigsten Erkrankungen für Betroffene und Interessierte. 2013, Montreux: Schweizerische Gesellschaft für Urologie.

8. Groeben, C., et al., Evaluation der Entscheidungshilfe Prostatakrebs aus Patientensicht. Ergebnisse der ersten 3 Monate. Urologe (Ausg. A), 2016. 55(12): p. 1586-1594.

9. International Organization for Standardization, ISO 9241-11:1998 - Ergonomic requirements for office work with visual display terminals (VDTs) - Part 11: Guidance on usability (1. Aufl.): 13.180; 35.180, 13.180; 35.180 (ISO 9241-11:1998). 1998.

10. Beckmann, I.-A., Prostatakrebs: Antworten, Hilfen, Perspektiven (Stand 2/2013). Die blauen Ratgeber: Bd. 17. 2013, Bonn: Dt. Krebshilfe.

11. Health_on_the_net_Foundation, The HON Code of Conduct for medical and health Web sites (HONcode). 2013.

12. Graham, I. and A. O'Connor. User Manual - Preparation for Decision Making Scale 19952014 10-05]; http://decisionaid.ohri.ca/docs/develop/User Manuals/UM PrepDM.pdf.

13. O'Connor, A. User Manual - Decisional Conflict Scale (16 item statement format). 1993 2014-1007]; http://decisionaid.ohri.ca/docs/develop/User Manuals/UM Decisional Conflict.pdf.

14. Boberg, E.W., et al., Assessing the unmet information, support and care delivery needs of men with prostate cancer. Patient Educ Couns, 2003. 49(3): p. 233-242.

15. O'Connor, A. User Manual - Decision Regret Scale. 1996 modified 2003 2014-10-15]; http://decisionaid.ohri.ca/docs/develop/User Manuals/UM Regret Scale.pdf.

16. Thompson-Leduc, P., et al., Prevalence of clinically significant decisional conflict: an analysis of five studies on decision-making in primary care. BMJ Open, 2016. 6(6).

17. Feldman-Stewart, D., et al., The Impact of Explicit Values Clarification Exercises in a Patient Decision Aid Emerges After the Decision Is Actually Made: Evidence From a Randomized Controlled Trial. Med Decis Making, 2012. 32(4): p. 616-626.

18. Chabrera, C., et al., A Decision Aid to Support Informed Choices for Patients Recently Diagnosed With Prostate Cancer: A Randomized Controlled Trial. Cancer Nurs, 2015. 38(3): p. E42-50.

19. Berry, D.L., et al., The Personal Patient Profile-Prostate decision support for men with localized prostate cancer: a multi-center randomized trial. Urol Oncol, 2013. 31(7): p. 1012-21.

20. Kaplan, A.L., et al., Decisional conflict in economically disadvantaged men with newly diagnosed prostate cancer: baseline results from a shared decision-making trial. Cancer, 2014. 120(17): p. 2721-7.

21. Stacey, D., et al., Decision aids for people facing health treatment or screening decisions. Cochrane Database Syst Rev, 2014(1): p. Cd001431. 